FRANCISCO JAVIER GONZÁLEZ MADARIAGA

UNIVERSIDAD DE GUADALAJARA

GUADALAJARA, MÉXICO

FRANCISCO.MADARIAGA@CUAAD.UDG.MX

JAIME FRANCISCO GÓMEZ GÓMEZ

UNIVERSIDAD DE GUADALAJARA

GUADALAJARA, MÉXICO

JAIME.GOMEZ@CUAAD.UDG.MX

LUIS ALBERTO ROSA SIERRA

UNIVERSIDAD PANAMERICANA

GUADALAJARA, MÉXICO

LUROSA@UP.EDU.MX
Financiamiento: Programa del Mejoramiento de Profesorado (PROMEP) del Gobierno Mexicano y a la Universidad de Guadalajara

Fecha de recepción: 29/08/2018

Fecha de aceptación: 24/09/2018

Cómo citar: González Madariaga, F., Gómez

Gómez, J. y Rosa Sierra, L. (2018) Placas

constructivas de menor impacto ambiental.

Avances de investigación. RChD: creación y

pensamiento, 3(5), 1-12

DOI: 10.5354/0719-837X.2018.49879

\section{Placas constructivas de menor impacto ambiental. Avances de investigación}

\author{
Environmentally friendly wallboard panels. Research report
}

Resumen. Se exponen avances del proyecto Placas constructivas de menor impacto ambiental que lleva a cabo el grupo de investigación en Innovación tecnológica para el diseño (CA 381), apoyada por la Universidad de Guadalajara, México. El proyecto se ocupa del diseño de nuevas placas para la construcción, útiles para fabricar particiones interiores o recubrir paramentos. El elemento constructivo se compone de: una parte central o núcleo, y un recubrimiento de papel en ambas caras del núcleo que se fabrica con un material compuesto de pastas de yeso y de dos residuos industriales; fibras secas de agave y partículas de plásticos expandidos. Los agregados tienen como objetivo mejorar la densidad y las propiedades de resistencia mecánica de las placas. A lo largo de la investigación se ha ensayado con más de setenta mezclas diferentes y muestras de las mismas se han sometido a pruebas en laboratorios de resistencia de materiales. Además, una de las hipótesis auxiliares de esta investigación señala que el nuevo material tiene un menor impacto al medio ambiente comparado contra otros similares. Para validar el cumplimiento de esta hipótesis se ha desarrollado una evaluación comparativa de ecodiseño, entre las nuevas placas contra productos similares ya disponibles en el mercado. En este documento se ofrece información resumida de los últimos resultados de laboratorio y de la evaluación de ecodiseño.

Palabras clave: nuevos elementos para construcción, reciclaje de plásticos, yeso.

\begin{abstract}
Here are shown some important research advances about the design process of a new building construction element, this is a wallboard flat panel manufacturated with a nucleus made of gypsum, agave fibers and water, mixed with different portions of EPS beads (Expanded polystyrene plastic). Natural fibers used here were obtained from a mexican plant called agave, the most important raw material in the tequila industry. Fibers and plastic particles are both recovered materials from the industrial waste flow and contribute to improve the wallboard's mechanical properties. Wallboards samples made of different mixtures were tested in labs and important research output is reported here. This project has been sponsored by the mexican government and carried out by the Tecnological Innovation for design, a research group from the University of Guadalajara, México. This paper focuses in output and data from the late research stages.
\end{abstract}

Keywords: building construction elements, gypsum plasters, plastics recovering

Revista Chilena de Diseño,

RChD: creación y pensamiento

Universidad de Chile

2018, 3(5)

http://rchd.uchile.cl 


\section{Introducción}

Los residuos no son nuevos para la humanidad, lo que los distingue hoy son las cantidades en que se encuentran, su origen, variedad de formas y corto ciclo de vida. Estas variables en conjunto provocan, por su difícil tratamiento, que algunos residuos se acumulen en nuestro entorno. Consideramos, además, que la presión social para gestionar rápidamente los residuos favorece la pérdida de la riqueza que aún guardan muchos de ellos al momento de ser desechados. Este proyecto se ha desarrollado de manera interdisciplinaria entre diseñadores industriales e ingenieros. Se busca proponer formas novedosas para el reciclaje de residuos y alargar su ciclo de vida, y así aprovechar las características que presentan algunos materiales al momento de ser dispuestos al flujo de residuos.

Para la fabricación de placas constructivas se propone el uso de materiales compuestos de corpúsculos de residuos de embalajes de plásticos expandidos, como el EPS (poliestireno expandido), y residuos de fibra seca de agave. Los plásticos y las fibras se unen con una matriz de pastas de yeso. El EPS es un plástico muy común en el flujo de residuos municipales y las fibras de agave son un abundante residuo de la fabricación de tequila, ambos materiales se mantienen unidos por una matriz de pastas de yeso y agua.

\section{Metodología y desarrollo}

El proyecto busca probar la hipótesis sobre la viabilidad del diseño en la producción de innovadores elementos planos para la construcción, fabricados con pastas de yeso, residuos de plástico y fibras de agave.

La metodología para este proyecto contempla labores de investigación documental y de campo, aunque privilegia el trabajo experimental. Se ordena en las siguientes etapas:

1. Investigación preliminar.

2. Propuesta de soluciones.

3. Experimentación.

4. Análisis de resultados.

5. Propuestas de aplicación.

\section{Etapa 1. Investigación preliminar}

Se determinó el problema a resolver: aprovechamiento de residuos de plásticos expandidos para fabricar elementos constructivos. En la etapa 1, se llevó a cabo una revisión de los aspectos fundamentales del problema, los cuales se dividen en elementos generales y elementos específicos. Los elementos generales atienden los conceptos básicos de la problemática de los residuos en el medioambiente, mientras los elementos específicos revisan información de carácter técnico y del estado del arte que guía el trabajo de desarrollo de un producto para la construcción con las características buscadas.

La investigación de los elementos generales ofrece algunas conclusiones importantes para el proyecto como:

- El consumo de plásticos se estimó (Plastics Europe, 2017) para el año 2015 en $322 \mathrm{Mt}$ (millones de toneladas sin aplicaciones no plásticas) del cual se recuperó con diversas técnicas y solo en algunos países cerca del $39 \%$. Esto dejó en los vertederos una importante cantidad anual (más de $196 \mathrm{Mt}$ ) de plásticos que se consideran residuos sin atender. De esto se puede extraer la urgencia en frenar la acumulación de residuos de plásticos. 
- La industria del reciclaje de plásticos se concentra en un pequeño grupo de materiales y deja sin atender muchos otros, como los plásticos celulares (SPI, 1999).

- Las técnicas de reciclaje comunes ofrecen polímeros industriales con propiedades disminuidas en comparación a los materiales vírgenes, lo que representa una desventaja importante para los materiales reciclados.

El proyecto tiene como uno de sus objetivos cooperar a la disminución de residuos de plástico y para ello considera tres estrategias: 1. por medio del mismo producto ya que podemos evitar o disminuir el uso de contaminantes en él; 2. promover tecnologías limpias para su producción; y 3. recuperar, reciclar y reutilizar los residuos industriales que pueden convertirse nuevamente en materia prima.

\section{Etapa 2. Propuesta de soluciones}

Con el análisis de la información preliminar de la etapa 1, se desarrollaron conclusiones que permitieron ofrecer propuestas de la aplicación de residuos de plásticos para fabricar elementos constructivos. Las ideas se evaluaron y depuraron en base a su viabilidad e importancia y donde resalta la oportunidad que hay en desarrollar dos productos para la construcción:

- Uno: placa constructiva de usos similares a la placa de yeso estándar. Las placas propuestas están fabricadas de varios tipos de conglomerantes de yeso, con inclusiones de residuos de espuma de EPS y recubiertas en su cara y dorso con cartón. Se les designa placa en esta investigación.

- Dos: un panel de aislamiento térmico para la construcción, de usos similares a los paneles de espuma virgen de EPS y que está formado por muy altas proporciones de residuos plásticos y pastas ricas en agua, a esa aplicación aquí se le designa como panel.

\section{Etapa 3. Experimentación}

La etapa 3 es la más importante del proyecto y se compone de:

3.1. Experimentación preliminar.

3.2. Experimentación general.

3.3. Evaluación de ecodiseño.

A partir de este punto y por razones de espacio, solo se exponen los principales resultados del ensayo a placas, y señalamos que los reportes completos de experimentación, que incluyen los resultados obtenidos de la experimentación con paneles, se ofrecen en otras publicaciones (González, 2006).

\section{Subetapa 3.1. Experimentación preliminar}

Se revisan los comportamientos de los residuos de espumas de plástico al mezclarse con pastas de yeso. También se ha experimentado sobre la forma y dimensiones que deben mostrar los residuos para su integración en las pastas que formarán placas y paneles.

Con base en la investigación preliminar, se diseñó un árbol de combinaciones de diferentes mezclas de tipos y proporciones de yeso, agua y residuos de EPS; y que organizados en grupos de trabajo sumaron sesenta y ocho fórmulas o mezclas, de las cuales se fabricó una muestra de cada una que fueron sometidas a ensayos simples, lo que permitió reducir la variedad de 
sesenta y ocho a solo ocho fórmulas de interés. De estas últimas, se fabricaron nuevas muestras, que fueron sometidas a cinco tipos de ensayos para su caracterización y evaluación: absorción de agua, densidad, flexotracción, y resistencia al impacto. Todas las muestras a ensayar se fabricaron con las medidas marcadas por la norma correspondiente (AENOR, 2001), $12.5 \times 300$ $\times 400 \mathrm{~mm}$ (Norma UNE 102035/1M:2001 Placa de yeso laminado). Algunas conclusiones del trabajo experimental preliminar de placas:

\subsubsection{Ensayo de absorción de agua por inmersión total de placa} Norma utilizada, UNE 102035/1M:2001 Placa de yeso laminado. Métodos de ensayo. Apartado 12(AENOR 2001).

El ensayo comprobó la tendencia a absorber más agua de los nuevos materiales, comparación con la absorción de las probetas sin plástico (>25\%) y las placas comerciales $(>2,5 \%)$. Es conveniente señalar que el retorno de agua absorbida hacia el exterior se da más rápidamente en los nuevos materiales, lo cual puede ser una ventaja en la mayor rapidez de recuperación en cuanto a las propiedades de resistencia.

3.1.2. Determinación de la masa por unidad de superficie Norma, UNE 102035/1M:2001 Placa de yeso laminado. Métodos de ensayo. Apartado 8 (AENOR 2001).

Los resultados han mostrado mejoras de densidad laminar que oscilan entre el $44,5 \%$ y el $23,1 \%$ entre los materiales que contienen residuos molidos de espuma y la placa de yeso estándar comercial.

\subsubsection{Resistencia mecánica a la flexión}

Norma, UNE 102035/1M:2001 Placa de yeso laminado. Métodos de ensayo. Punto 9 (AENOR 2001).

El ensayo evidenció la disminución de los valores de carga máxima a la flexión que experimentan los cuerpos de yeso cuando se han formulado con una alta proporción de agua o se les ha agregado residuos molidos de EPS, $\mathrm{O}$ cuando suceden los dos casos.

\subsubsection{Resistencia al impacto}

Norma, UNE 102035/1M:2001 Placa de yeso laminado. Métodos de ensayo. Apartado 10 (AENOR 2001).

De la comparación de materiales ensayados, tres grupos de materiales, los formulados con residuos de EPS son apenas más débiles al impacto.

La prueba, que consistió en la medición de la huella producida por la caída libre de una bola de acero sobre la cara de la muestra, señaló que las mezclas con residuos de EPS mostraron huellas que exceden hasta en un $15 \%$ a los diámetros permitidos en la Norma.

\subsubsection{Algunas conclusiones de la etapa 3.1}

Entre las conclusiones más importantes que resultan de la etapa de experimentación preliminar son:

- Se confirmó que la recuperación de plásticos expandidos ofrece una oportunidad para cooperar en la mejora del medioambiente.

- La mejor forma de aprovechamiento de los residuos de plásticos expandidos, para los productos que se buscan, es la separación en cuerpos individuales (perlas) que conforman los plásticos expandidos. 
- Se requiere la mejora en las respuestas a esfuerzos de flexotracción de las placas ensayadas.

Con base en la experimentación que se describe en el punto 3.1., se comprobó que es viable la aplicación de materiales compuestos que involucren el uso de residuos de expandidos de plástico mezclados con otros materiales para la fabricación de elementos constructivos.

\section{Subetapa 3.2. Experimentación general}

Los resultados obtenidos en los ensayos de la etapa 3.1. alentaron la continuación de la investigación, donde uno de los retos para la mejora de las mezclas fue aumentar su resistencia a la flexotracción; para cumplir con esta exigencia se estudiaron candidatos de fibras de refuerzo y finalmente se optó por adicionar a las mezclas con fibra seca de agave tequilana weber, estas fibras son un residuo abundante que procede de la planta de maguey de esa variedad y que es base de la industria del tequila, importante bebida alcohólica fabricada con denominación de origen en varias zonas de México.

Objetivos de la etapa:

a. Determinar las proporciones adecuadas de los componentes del (los) nuevo(s) material(es) para ser usados en la fabricación de placas para la construcción.

b. Valorar la mejora que ofrece fibra seca de agave a las mezclas.

c. Caracterizar los materiales con que se fabricarán los elementos constructivos propuestos.

Para la subetapa 3.2 se prepararon dos nuevos grupos de muestras, el primero siguiendo las 8 fórmulas de la subetapa 3.1, pero ahora enriquecidas con diferentes cantidades de fibra de agave. Estas muestras de placas fueron sometidas a los mismos ensayos de laboratorio. De los mejores resultados de los ensayos se seleccionaron dos mezclas, y se prepararon nuevas muestras ahora sólo de estas dos fórmulas. Se denominaron mezclas 121 y 123 respectivamente. Su composición se expone en la Tabla 1.

Fórmula Yeso fino(\%) Yeso Vulgar(\%) Agua(\%) EPS(\%) Fibra de agave(\%)

\begin{tabular}{|c|c|c|c|c|c|}
\hline 121 & 35 & 23.3 & 39.00 & 0.95 & 1.75 \\
\hline 123 & 42 & 17.8 & 37.45 & 0.95 & 1.80 \\
\hline
\end{tabular}

En todas las muestras se toma el 100\% de yeso para las relaciones de la fórmula, esto es: todas las cantidades mostradas en agua, EPS y fibra seca de agave surgen en relación a la cantidad en peso de yeso. En la Tabla 1, se muestra de manera general la nomenclatura empleada en la etapa II, se omiten las proporciones.

El nuevo grupo de muestras (como las usadas en la etapa I) se fabricaron por vaciado en moldes abiertos, así las placas ofrecen una cara suave y otra rugosa; se observaron imperfecciones en el dorso de algunas muestras lo que se puede atribuir a la presencia de impurezas del conglomerante, a la flotación de perlas de residuo de EPS en las capas superiores del núcleo de yeso, y a la distribución heterogénea de residuos de fibra de agave en las muestras (González, 2008).
Tabla 1. Fórmulas utilizadas para la fabricación de muestras. concentración porcentual en peso (g) 
Gráfico 1. Porcentaje de absorción de agua y pérdida posterior. Fuente: elaboración propia

Tabla 2. Masa por unidad de superficie promedio por tipo de Fórmula

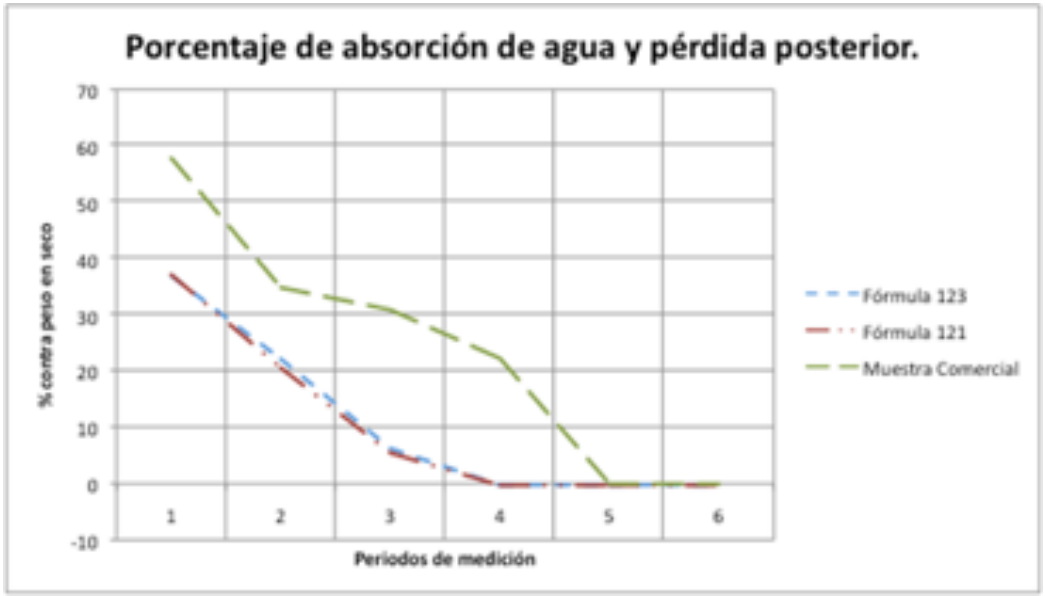

Los ensayos practicados a las nuevas muestras son los mismos que aquellos de la etapa I, esto permite establecer una comparación entre ambos trabajos de laboratorio. Los resultados del trabajo experimental se resumen a continuación:

\subsubsection{Ensayo de absorción al agua por inmersión total de las placas} Norma UNE 102035/1M:2001 Placa de yeso laminado. Métodos de ensayo. Apartado 12. Capacidad de absorción de agua. Determinación de absorción total de agua (AENOR, 2001).

De los datos obtenidos, podemos decir que las muestras que solo contienen en su composición EPS, agua y yeso, tienden a absorber una mayor cantidad de agua en comparación a las placas que contienen fibra de agave, sin embargo, el agua absorbida por esas últimas es desechada en un período más corto. En promedio, las placas con los componentes de yeso, EPS y fibra de agave reportan un aumento de peso aproximado al 40\% mayor, en comparación al peso presentado 10 minutos después de su escurrimiento. Mientras que la muestra comercial presentó un aumento de $58 \%$. Este aumento de peso disminuye paulatinamente y en diferentes cantidades, hasta recuperar el peso original de cada muestra, pero es notoria la mayor velocidad con la que la humedad se pierde en las muestras fabricadas con los nuevos materiales que incluyen fibras de agave. Ver Gráfico 1.

\subsubsection{Ensayo de Densidad Laminar}

Norma UNE 102035/1M:2001 Placa de yeso laminado. Métodos de ensayo.

Apartado 8. Determinación de la masa por unidad de superficie (AENOR, 2001). La densidad laminar es la cantidad total de la masa de una placa en función a su espesor expresado en $(\mathrm{kg} / \mathrm{m} 2)$. Se puede concluir que la composición que se necesita para obtener los resultados deseables para el proyecto proviene de un balance entre ambos grupos: si bien, el EPS disminuye la densidad de los nuevos materiales hasta en un $\mathbf{2 6} \%$, el uso de fibra en placas afecta negativamente esa ventaja, como se observa en la Tabla 2.

\begin{tabular}{|c|c|}
\hline Fórmula & Masa de la mezcla ensayada $\left(\mathrm{kg} / \mathrm{m}^{2}\right)$ \\
\hline comercial & $8.5-10$ \\
\hline 121 & 9.95 \\
\hline 123 & 9.61 \\
\hline
\end{tabular}



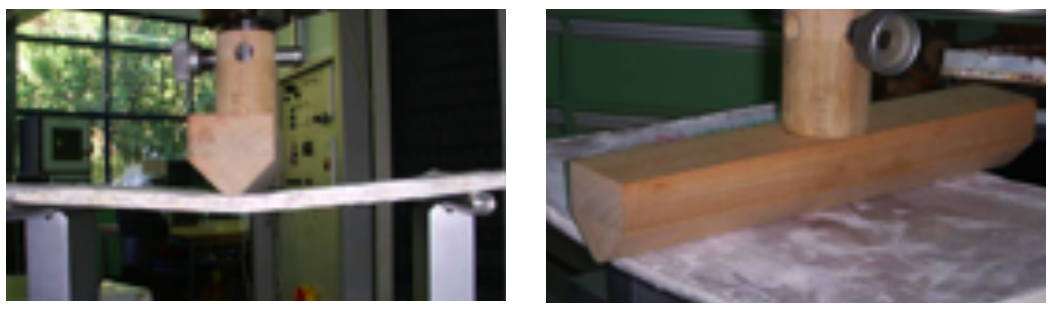

\begin{tabular}{|c|c|c|}
\hline Fórmula & Carga máxima de rotura (N) & Deformación (milímetros) \\
\hline 123 & 320.25 & 0.62875 \\
\hline 121 & 347.37 & 0.6075 \\
\hline comercial & 253.5 & 0.835 \\
\hline
\end{tabular}
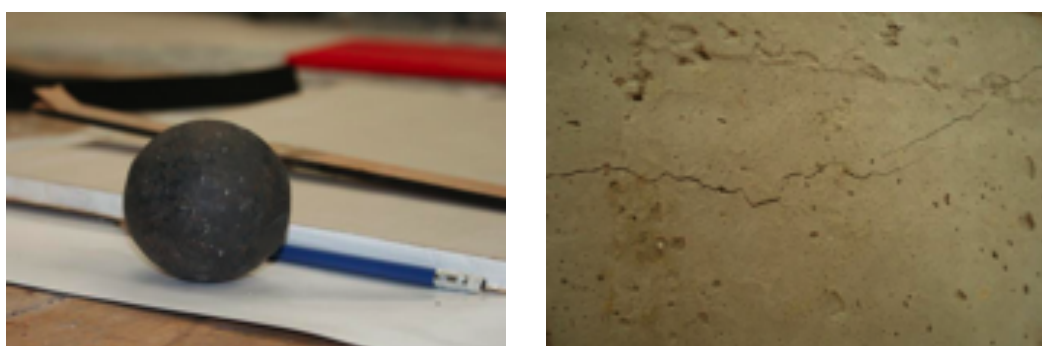

\subsubsection{Ensayo de Flexión de las placas}

Norma UNE 102035/1M:2001 Placas de yeso laminado, métodos de ensayo.

Punto 9. Resistencia mecánica a la flexión (AENOR, 2001).

El ensayo se aplicó en una máquina universal de pruebas (máquina modelo Karl Frank- MOD- $8150000 \mathrm{~N} 5$ Toneladas, 0.5 mm/min.) que tiene un desplazamiento vertical. El banco de pruebas instalado en la platina móvil de la máquina soporta la probeta y la eleva presionando contra un rodillo fijo hasta que se produce la fractura (Figuras 1 y 2 ).

Se reporta que el grupo de placas con agregados de fibra de agave presentó una resistencia mayor a la flexotracción, esto parece confirmar que los agregados de fibra de agave favorecen la resistencia a este tipo de esfuerzos. La Tabla 3 detalla los resultados.

\subsubsection{Ensayo de Resistencia al impacto de las placas}

El ensayo se ha adecuado en lo señalado en la Norma UNE 102035/1M:2001 Placa de yeso laminado. Apartado 10. Resistencia al impacto (AENOR, 2001). Se busca determinar el diámetro de la huella que genera una esfera de acero al momento de impactarse en la superficie de la placa con una energía potencial de $245 \mathrm{~J}$. Esta energía se consiguió a través de la caída libre de una esfera de acero desde una altura de $0.21 \mathrm{~m}$ sobre una de las caras de cada muestra. Ver figuras 3 y 4 .

\subsubsection{Algunas conclusiones de la etapa}

Se ofrecen aquí algunas conclusiones de importancia que resultan de los ensayos practicados en la etapa 3.2:

De los resultados de experimentación, se puede inferir que la fibra de agave aporta mejoras a las propiedades de resistencia al impacto en las muestras
Figura 1 y 2. Aplicación de ensayo a flexotracción de muestras de placas. Fuente: autores

Tabla 3. Carga máxima de rotura a la flexión y deformación promedio por tipo de fórmula

Figura 3. Bola de acero usada en el ensayo. Fuente: elaboración propia Figura 4. Registro del impacto Fuente: elaboración propia 
donde participa, por lo que se considera positiva su inclusión en las mezclas. Se observa una mejor resistencia a la flexotracción de las placas que contienen plásticos y fibras, en comparación de las placas disponibles en el mercado. En los resultados de los ensayos en comportamientos de especial interés, como la resistencia al impacto, se observó que solo un grupo de placas superó los límites máximos (diámetros $\leq 20 \mathrm{MM}$ ) permitidos por la Norma, este fue el grupo de mezclas que contienen yeso fino. En la mayoría de los casos, las placas que contienen fibra de agave exhiben resultados favorables donde se presentaron huellas con medidas de áreas menores a las exigidas por la normatividad para placas constructivas (González, 2012).

\subsection{El ecodiseño}

En esta subetapa se examina, a través de una evaluación de ecodiseño, el cumplimiento de la hipótesis que señala una disminución del impacto al medioambiente de los nuevos materiales, comparada con algunos productos similares empleados en la industria de la construcción.

Bajo el nombre genérico de ecodiseño se engloban diversos términos y filosofías que son de gran importancia para el cumplimiento de los objetivos del proyecto que nos ocupa: promoción de productos mejorados desde el punto de vista del medioambiente.

Hay coincidencia en los investigadores alrededor del ecodiseño, y esta es que se trata de considerar los aspectos del medioambiente que se relacionan con un producto a lo largo de todo su ciclo de vida, al tiempo que se busca que las funciones propias del producto sean las más eficientes. La mayoría de los objetivos buscados por el ecodiseño solo se pueden lograr si son incorporados al proyecto durante las primeras etapas en la concepción del producto, esto es durante la fase de diseño y desarrollo de producto (Rieradevall, 1999).

Disminuir el impacto al medioambiente es uno de los principales objetivos de este proyecto, así se ha explorado a través de una evaluación de ecodiseño el comportamiento ambiental de las muestras, comparadas contra algunos ejemplos de productos comerciales similares. Para la evaluación se empleó la metodología propuesta por IHOBE (1999). Los resultados completos se publicarán prontamente, mientras que acá se expondrán los resultados más relevantes.

La aplicación del ecodiseño es una condición que consideramos imprescindible hacia el diseño y desarrollo de productos de menor impacto al medioambiente. El equipo de diseño de producto puede cooperar a la sostenibilidad a través de la aplicación secuenciada y sistemática de algunas de las herramientas del ecodiseño. En el resumen de la evaluación de ecodiseño que se expone, se aplican diversas herramientas de recolección de información y análisis, los resultados son especialmente útiles en la toma de decisiones durante el proceso de diseño. Se enfatiza en la disminución de los requerimientos de energía y materiales para la fabricación, así como el manejo de residuos durante todas las etapas del ciclo de vida de un producto de diseño: placas de yeso adicionadas con partículas de plástico expandido y fibras de agave, donde estos dos últimos materiales proceden del flujo de residuos.

\subsubsection{Etapas del proceso de ecodiseño}

Las etapas generales del proceso de ecodiseño empleado aquí son:

- Creación del equipo y planificación.

- Evaluación de ecodiseño. 
- Implantación de las mejoras determinadas en la evaluación.

- Seguimiento de las mejoras.

- Valoración del proyecto.

Acá solo se resumen los trabajos desarrollados en la etapa de Evaluación de Ecodiseño (en adelante EEC), de los cuales se hace una breve descripción.

a. Descripción del producto.

b. Descripción de materiales. La identificación precisa de los materiales con los que está fabricado el producto por evaluar son por lo general una fuente potencial de mejoras.

c. Descripción del sistema producto. Esta etapa relaciona las cinco etapas del ciclo de vida del producto con los materiales y procesos involucrados.

d. Determinación de la unidad funcional. De acuerdo a los intereses del proyecto se establece una cantidad de producto a evaluar.

e. Aplicación de la matriz MET. La matriz MET es una herramienta semicuantitativa de análisis de impacto medioambiental de producto, la que permite organizar de manera sistemática la información ambiental relevante relacionada con la evaluación, lo que facilita al equipo de ecodiseño la posibilidad identificar amenazas y oportunidades de mejora. La matriz MET permite capturar y organizar en una tabla, información acerca de los materiales (M) utilizados, la energía (E) consumida y las emisiones ( $T$ ) generadas durante las diferentes etapas del ciclo de vida de un producto.

f. Análisis de la matriz MET.

g. Ecoperfil de producto. Al usar la información obtenida, el equipo de ecodiseño evalúa el desempeño actual del producto. La gráfica está compuesta por seis vectores que son: 1. satisfacción de las funciones que debe cumplir el producto; 2. eficiencia de la selección de materiales en los que está fabricado; 3. fabricación del producto, eficiencia en la selección y aplicación de las técnicas de producción a través de las cuales se obtiene el producto; 4.comercialización y distribución, descripción y evaluación del proceso que lleva el producto evaluado de su fabricante al usuario del mismo; 5. uso del producto, el equipo evalúa cómo se usa el producto e identifica oportunidades de mejora; 6. eliminación final o disposición, la forma como el producto finaliza su ciclo de vida es también una fuente de oportunidades de mejora. La evaluación se practica por el equipo mediante una calificación asociada a la satisfacción que ofrece el producto en los seis vectores, finalmente los resultados se unen formando una gráfica poligonal.

h. Aplicación de ecoindicadores. Los materiales identificados en el punto de descripción de materiales se relacionan ahora con las cantidades necesarias de ellos para satisfacer la unidad funcional, a través de ecoindicadores que son valores adimensionales que cuantifican el impacto ambiental de un material y/o proceso de producción específicos.

i. Tablas de ecoindicadores, producción, uso y disposición. En una tabla se relacionan las cantidades obtenidas para cumplir con la unidad funcional con el ecoindicador correspondiente, de aquí se obtiene un indicador de desempeño ambiental expresado en Mp (milipuntos); el resultado es un elemento de comparación útil de una estrategia o acción de diseño con su desempeño medioambiental.

j. Propuestas de mejora. Con base en la información obtenida, el equipo de diseño propone estrategias precisas para mejorar el producto, relacionándolas con cada etapa de su ciclo de vida. 
Tabla 4. Resultados de evaluación de ecodiseño (Puntos)
22 P.

13.7 P.

13.3 P.

k. Viabilidad de las mejoras. El equipo de ecodiseño califica las propuestas del punto anterior con criterios como: sencillez técnica, factibilidad económica y otros de importancia particular para la estrategia que se pretende aplicar.

I. Propuesta de alternativas de mejora. Se comparan los desempeños actuales de los productos evaluados con los resultados hipotéticos de la aplicación de alternativas propuestas.

$m$. Ecoperfil comparativo de resultados de ecodiseño. Al retomar la gráfica poligonal, se evalúan y comparan los resultados obtenidos de la aplicación de las alternativas de mejora al producto, con relación al estado inicial del proyecto.

\section{Desarrollo de la evaluación}

Una vez determinadas y caracterizadas las nuevas placas constructivas, se practicó una evaluación comparativa ECc entre dos mezclas de las ensayadas (mezclas 121 y 123) contra otra placa similar disponible en el mercado. La evaluación tiene como objetivo principal validar las mejores conductas medioambientales de los nuevos productos.

\section{Resultados}

La información que ha arrojado la evaluación está todavía en revisión, aunque partes de los resultados se han publicado (Hernández, 2016). Una parte importante del procedimiento se basó en la metodología marcada por el ıнове que señala cinco etapas del ciclo de vida a revisar: obtención de materiales, producción, comercialización y distribución, uso, y fin de vida. Los resultados se resumen en la Tabla 4.

\section{Conclusiones de la evaluación}

Los resultados de la evaluación obtenidos tienden a evidenciar un mejor comportamiento ambiental de las placas formuladas con pastas de yeso, partículas de plástico y fibra de agave (13.7 y 13.3 P), comparados con los resultados obtenidos por las placas comerciales (22 P).

Se puede concluir de la evaluación que los principales impactos al medio se encuentran en la obtención y procesado de los componentes, seguidos por su distribución y uso, mientras que la etapa de fin de vida reporta los menores impactos.

\section{Conclusiones}

De las aplicaciones de los materiales que contienen yeso, EPS y fibra de agave, en elementos de uso similar a las placas de yeso estándar:

Una ventaja de los nuevos materiales de placa está en su peso competitivo, donde una placa de $12,5 \mathrm{~mm}$ de uno de los nuevos materiales tiene un peso que va desde $5,55 \mathrm{~kg} / \mathrm{m} 2$ hasta $10 \mathrm{~kg} / \mathrm{m} 2$, mientras una placa comercial reporta un peso que oscila entre 9,8 y $10 \mathrm{~kg} / \mathrm{m} 2$. Sin embargo, esta ventaja se ve comprometida al agregar fibras de agave en la fórmula de las muestras. El hecho de mantener una menor densidad en los materiales constructivos que se ensayan puede traducirse en un beneficio medioambiental y que se añade al hecho del uso de materiales reciclados. 
Los ensayos han confirmado las buenas características de reacción al fuego de los materiales propuestos, lo que ofrece una opción válida y atractiva para aplicaciones de construcción interior con mayor riesgo de incendio y donde la placa de yeso estándar puede resultar más pesada.

En varias de las comparaciones entre las resistencias mecánicas se observa un mejor desempeño de los materiales comerciales contra las placas que no contienen fibra. Las placas que contienen fibra tienen una resistencia mayor a la flexotracción, de $21 \%$ de la muestra 123 , y de $28 \%$ de la muestra 121 . Del grupo de ensayos aplicados a muestras, se confirma que los nuevos materiales son competitivos contra las placas estándar.

Se ha mostrado que las aplicaciones propuestas son una alternativa para el reciclaje de residuos de espumas de EPS y para disminuir los volúmenes o postergar la llegada de las espumas a los vertederos.

Se ha comprobado que los materiales que contienen residuos acondicionados de EPS y fibra de agave, pueden ser aprovechados para la fabricación de elementos constructivos tipo placa, como los que se han señalado a lo largo de la investigación.

Se ha logrado la propuesta de productos alternativos para la construcción que pueden fabricarse con procesos simples.

Se ha logrado la protección intelectual de algunas mezclas ensayadas mediante la obtención de la patente ES $2277776 \mathrm{Bl}$.

Se ha logrado presentar una propuesta de diseño industrial mediante la realización de un trabajo multidisciplinario de diseñadores e ingenieros, que busca cooperar a la búsqueda de soluciones a problemas sociales, como es en este caso, el de los residuos urbanos como los plásticos expandidos y de residuos industriales como la fibra de agave.

Podemos observar que los resultados obtenidos en ambas etapas experimentales ayudan a confirmar la viabilidad del proyecto, ya que muestra datos con pocas diferencias en comparación a los productos encontrados actualmente en el mercado.

Está clara la necesidad de hacer ajustes que ayuden a mejorar la calidad de las nuevas placas y que además mejoren detalles en aspectos de fabricación, pero estos avances han sido fundamentales en el crecimiento del proyecto debido a que en cada etapa se obtuvo resultados con diferencias a los anteriores por la inclusión de nuevos residuos.

\section{Agradecimientos}

Al Programa del Mejoramiento del Profesorado (PROMEP) del Gobierno Mexicano y a la Universidad de Guadalajara. Por el financiamiento y apoyo para el desarrollo de este proyecto.

\section{Asistentes investigadores}

Ing. Zaira Selene del Sordo Nuñez. UAS. México, Ing. Ana Patricia González Quiñonez, L.D.I. José Luis Rubén García UDG. México, L.D.I. Xitlalli Mayahuel Sepúlveda A. UDG. México, D.I. Moisés Carrillo Joya uDG. México, Mariel Andrea Ojeda Tuz. Universidad Autónoma de Yucatán, Paulina Elena Romero Laureano. (UDG) 


\section{Referencias}

González Madariaga, F.J. (2006) Caracterización de mezclas de residuos de poliestireno expandido conglomerados con yeso o escayola, su uso en la construcción. Tesis doctoral. UPC, ETSEIB. Barcelona (2006) ISBN 978-84-690-6065-O URL <https://upcommons.upc.edu/handle/2117/94156> AENOR. (2001) Norma UNE 102035:2001. Placas de yeso laminado. Métodos de ensayo. Madrid AENOR.

SPI. (1999) Guide for standard worlwide terminology for use in recycling of plastics. The society of Plastics Industry, Inc. Reproducida por ANARPLA. Valencia España

Plastics Europe (2017). The facts. Association of plastics manufacturers. Brussels. https://www.plasticseurope.org/application/ files/5715/1717/4180/Plastics_the_facts_2017_FINAL_ for_website_one_page.pdf

González Madariaga, F.J. (2006) Caracterización de mezclas de residuos de poliestireno expandido conglomerados con yeso o escayola, su uso en la construcción. Tesis doctoral. UPC, ETSEIB. Barcelona (2006) ISBN 978-84-690-6065-0 URL <https://upcommons.upc.edu/handle/2117/94156> Rieradevall, J.; Vinyets, J. (1999) Ecodisseny i ecoproductes. Barcelona: Rubes Editorial,S.L. 1a. ed.

IHOBE (1999). Anexo. Eco-indicator '99. Soc. Pública Gestión Medioambiental. Goedkoop et. al Bilbao González Madariaga F.J, Lloveras Macià, J. (2008) EPS recycling bends mixed with plastic or stucco, some applications in building construction. Informes de la construcción. Instituto de Ciencias en la construcción Eduardo Torroja, Madrid. Issn: 0020-0883

González Madariaga F.J. Rosa Sierra L.A. (2012) Caracterización de mezclas de residuos de poliestireno expandido (EPS) conglomerados con yeso, su uso en la construcción. Reporte de investigación 2008 - 2016. CUAAD. Universidad de Guadalajara, Guadalajara Jalisco, México.

Hernandis O. B.(compilador) 6th. International Forum of Design as a Process System \& Design. Universitat Politecnica de Valencia. 2016. pp 903.

DOI: http://dx.doi.org/10.4995/IFDP.2016 